\title{
Análisis del rendimiento de los protocolos OLSR, DSDV y AODV en una Red MANET
}

\section{Performance analysis of OLSR, DSDV and AODV protocols in a MANET Network}

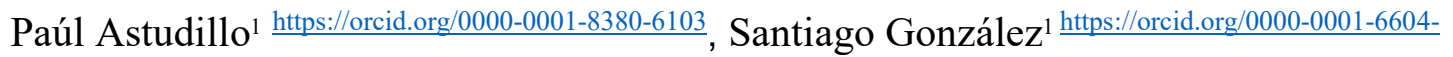 \\ 889X , Christian Quinde1 https://orcid.org/0000-0002-9863-6941
}

\author{
${ }^{1}$ Departamento de Eléctrica, Electrónica y Telecomunicaciones, Universidad de \\ Cuenca, Cuenca, Ecuador \\ paul.astudillop@ucuenca.edu.ec, \\ santiago.gonzalezmaucuenca.edu.ec, \\ christian.quinde@ucuenca.edu.ec
}

\section{(c) $(1) \Theta$}

Esta obra está bajo una licencia internacional

Creative Commons Atribución-NoComercial 4.0. $\begin{array}{ll}\text { Enviado: } & 2021 / 06 / 24 \\ \text { Aceptado: } & 2021 / 09 / 28 \\ \text { Publicado: } & 2021 / 11 / 30\end{array}$

\section{Resumen}

Este artículo presenta una propuesta de análisis y comparación entre los protocolos de enrutamiento proactivo OLSR y DSDV, y reactivo AODV en una red MANET. Se definen dos escenarios: el primero representando un equipo de fútbol atacando y el segundo defendiendo. Se varía el área de movimiento de los nodos para simular cada escenario. Para ello, se realiza una investigación sobre los problemas que presentan estos algoritmos de enrutamiento para redes Ad Hoc en diferentes ámbitos. De esta forma, obtener un panorama de las estrategias y soluciones que existen actualmente. A partir de la información recopilada, se propone realizar una simulación utilizando NS3, para obtener resultados que se asemejen a la realidad. La comparación se realiza a base de las métricas de throughput, PDR y Delay. En cada simulación, la velocidad de los nodos y la posición inicial se ajustan según el comportamiento de un jugador de fútbol real. Los nodos se mueven en una trayectoria aleatoria dentro del área específica, y uno de éstos envía datos a un nodo fijo situado en el borde del campo. El tráfico transmitido está caracterizado para simular datos biométricos realistas. A partir de los resultados, se concluye que AODV y DSDV tienen un mejor rendimiento que OLSR y el intervalo óptimo de transmisión es de 20 segundos. Además, con una potencia de transmisión de $10 \mathrm{dBm}$ se garantiza el $100 \%$ del throughput en el receptor.

Palabras clave: delay, NS3, mapa de calor, PDR, throughput.

Sumario: Introducción, Trabajos relacionados, Materiales y Métodos, Resultados y Discusión y Conclusiones.

Como citar: Astudillo, P., González, S. \& Quinde, C. (2021). Análisis del rendimiento de los protocolos OLSR, DSDV y AODV en una Red MANET. Revista Tecnológica - Espol, 33(2), 43-57.

http://www.rte.espol.edu.ec/index.php/tecnologica/article/view/829 


\begin{abstract}
This paper presents an analysis and comparison between the proactive routing protocols OLSR and DSDV and the reactive routing protocol AODV in a MANET network. Two scenarios were defined, the first one representing a soccer team attacking and the second one defending. To simulate each scenario the area of movement of the nodes (players) was varied. For this purpose, an investigation was carried out on the problems presented by these routing algorithms for Ad Hoc networks in different environments. In this way, an overview of the strategies and solutions that currently exist is obtained. Based on the information gathered, it is proposed to perform a simulation using NS3 in order to obtain results that resemble reality. The comparison is made based on throughput, PDR and Delay metrics. In each simulation, parameters such as node velocity and initial position are adjusted according to the behaviour of a real soccer player. The nodes move in a random trajectory within the specific area and one of them sends data to a fixed server node located at the edge of the area where the nodes move, simulating the bench where the receiver is located. The transmitted traffic is characterized to simulate realistic biometric data. From the results, it is concluded that AODV and DSDV have better performance than OLSR and the optimum transmission interval is 20 seconds. In addition, with a transmit power of $10 \mathrm{dBm}, 100 \%$ throughput is guaranteed at the receiver.
\end{abstract}

Keywords: delay, heatmap, NS3, PDR, throughput.

\title{
Introducción
}

Una red ad hoc móvil (MANET) es un tipo de sistema distribuido complejo que comprende de nodos móviles inalámbricos que puedan autoorganizarse dinámicamente en topologías de red ad hoc temporales. Esto permite a las personas o dispositivos trabajar en Internet en áreas sin infraestructura de comunicación preexistente (Kurniawan et al., 2020). En las redes ad hoc, los nodos no están familiarizados con la topología de sus redes, por el contrario, tienen que descubrirlo. Normalmente, un nuevo nodo anuncia su presencia y escucha los mensajes transmitidos por sus vecinos. Cada nodo aprende acerca de otros cercanos y cómo llegar a ellos. Para esto existen tres tipos de protocolos: Proactivos, Reactivos e Híbridos. Este artículo se centra únicamente en los dos primeros. DSDV y OLSR son protocolos proactivos basados en tablas de ruteo que se actualizan de forma regular. Por el contrario AODV es un protocolo reactivo que trabaja a petición para crear nuevas rutas (Bai et al., 2017).

Hoy en día en el deporte, la tecnología ha tomado un papel muy importante y el fútbol no es la excepción. La introducción de la tecnología dentro del deporte ha permitido una mejora en la formación de los atletas. Dentro de los entrenamientos, existen ejercicios que demandan un mayor esfuerzo físico, es el caso del sprint, que es un ejercicio de alta intensidad donde los atletas llegan a su máxima capacidad. Por lo tanto, resulta indispensable realizar una medición de los datos físicos del atleta o jugador (Lamaarti et al., 2019). En este contexto es necesario tener en cuenta métricas como el throughput, el delay end-to-end, el jitter y el PDR. Y se debe priorizar alguna para mejorar la calidad de servicio (QoS). Aquí es donde toma importancia el protocolo de enrutamiento utilizado en la red. Dentro de los inconvenientes que surgen en las redes MANET, están el área de interferencia entre nodos, la distancia entre los mismos y la velocidad de desplazamiento de los nodos. Todos estos factores influyen directamente en las métricas mencionadas anteriormente. Por tal razón, en este artículo se utilizan protocolos que tienen una base sólida, que han sido probados y se ha demostrado que son robustos y eficientes.

La simulación sigue desempeñando un papel vital en términos de escalabilidad tanto en tamaño como en velocidad experimental. Con estudios basados en simulación, el enfoque se puede analizar en detalle a diferentes escalas, con diversas aplicaciones de datos, diferentes condiciones de campo, con el fin de obtener resultados reproducibles y analizables. De ahí que, 
se propone el uso de NS3 junto con el módulo Flow Monitor para realizar la simulación. NS3 es un software ampliamente empleado para investigación, ya que es un programa muy realista, los modelos que usa están apegados a la realidad, se puede utilizar semillas para generar aleatoriedad y obtener resultados estadísticos aceptables (Campanile et al., 2020). Además, al trabajar con un gran número de nodos, diferentes tipos de movilidad, entre otros parámetros, el software de simulación es una herramienta que proporciona ventajas, debido a las limitaciones que implica trabajar en escenarios con una gran cantidad de nodos en la realidad.

En este artículo se presenta una evaluación de los protocolos de enrutamiento OLSR, AODV y DSDV para el despliegue de una red MANET enfocada en la transmisión de datos biométricos. En particular, se ha seleccionado el caso de un escenario deportivo con nodos móviles. En la sección 2 se presentan los trabajos relacionados a los protocolos de enrutamiento en redes Ad-Hoc, así como de sensores biométricos en ambientes deportivos. Adicionalmente, en la sección 3 se encuentra la metodología empleada y las herramientas utilizadas. En la sección 4 se presentan los resultados obtenidos y finalmente, en la sección 5 se encuentran las conclusiones.

\section{Trabajos relacionados}

De acuerdo a (Sharma \& Shruti Thapar, n.d.), se deben tomar en cuenta las características específicas de cada aplicación para escoger un protocolo sobre otro. También se detalla que los protocolos reactivos tienen un mejor rendimiento que los proactivos en un escenario con movilidad, no así para un escenario fijo o estático. En (Gamess \& Russoniello, 2018; Singh \& Verma, 2015) se señala que el protocolo de enrutamiento proactivo OLSR presenta un menor delay end-to-end y menor número de saltos comparado con DSDV. En (Yefa Mai et al., 2017) se demuestra que AODV es mejor en cuanto al throughput y delay endto-end, pero OLSR es mejor en la entrega de paquetes. Al aumentar el área de movilidad, el protocolo AODV se vuelve dominante en todas las métricas. De manera similar en (Singh \& Verma, 2015), se comprueba que AODV es mejor que OLSR y DSDV en condiciones de nodos móviles, pero con nodos fijos OLSR supera a AODV y a DSDV en las métricas de throughput y PDR. Además en (Al-Dhief et al., 2018) se establece que AODV supera a DSDV en términos de throughput.

En el ámbito deportivo existen varios estudios del movimiento en atletas, por ejemplo, en (Massard et al., 2018) se realiza un análisis de la velocidad máxima de jugadores de fútbol. Asimismo en (Modric et al., 2020) se obtienen valores para la velocidad de desplazamiento dependiendo de la posición de juego, así como del estado físico de cada jugador. De igual manera, en el estudio que realiza (Wehbe et al., 2014), se obtiene el tiempo para cada movimiento que efectúa un jugador de acuerdo a su posición. Los perfiles de actividad física de los jugadores revelan una información valiosa para conocer el esfuerzo físico que realiza el jugador y así mantenerlo en un estado óptimo, evitar lesiones y desarrollar nuevas estrategias de acuerdo al posicionamiento que se observa en los mapas de calor. Los entrenadores están interesados en herramientas analíticas para apoyar el análisis táctico y controlar la calidad de sus jugadores durante partidos individuales o temporadas enteras. Debido a esto, existen varios estudios donde se analiza la posición de cada jugador en el campo de juego de acuerdo a su rol (Defensa, Medio-campista, Delantero, etc.) (Pappalardo et al., 2019). Al tener un perfil de actividad física de cada jugador se puede limitar su movimiento con la ayuda de los mapas de calor de acuerdo a su posición. De esto depende la formación usada por el equipo.

En (Akbar et al., 2016) se mencionan diferentes tipos de sensores biométricos, como por ejemplo un medidor del ritmo cardíaco, un sensor SpO2, Acelerómetro, Giroscopio, etc. Cada uno de estos sensores genera información acerca de la actividad física. Según (Lloret et 
al., 2016), la cantidad de información útil (payload) de un paquete con información de salud puede alcanzar un tamaño promedio de 104 bytes. Asimismo, en (Besher et al., 2020) y (Beitelspacher et al., 2020), se muestra que el tamaño de un paquete de datos biométricos es de 79 bytes y 50 bytes respectivamente, las diferencias se dan debido al número de sensores, tipo de sensores y frecuencia de recolección de datos.

Otro aspecto fundamental para caracterizar el tráfico y mejorar los resultados de la simulación, es el data rate. En (Akbar et al., 2016) y (Kos et al., 2019), se muestran los requerimientos para aplicaciones médicas. Donde se encuentran los valores óptimos para el data rate (ver, Tabla 1) además el delay y el consumo de energía para algunos tipos de sensores usados en el deporte. Es de vital importancia el monitoreo de la salud dentro del deporte, en (R. T. Li et al., 2016) se señala que los avances en la tecnología han permitido a los atletas de resistencia individuales, equipos deportivos y médicos, monitorear los movimientos funcionales, cargas de trabajo y marcadores biométricos para maximizar el rendimiento y minimizar las lesiones. Con la misma idea en ( $\mathrm{S}$. Li et al., 2020) se señala que los sensores corporales en el campo deportivo tienen un gran impacto en la eficiencia de la vida, ya que estos se enfocan en medir factores fisiológicos, promover la salud y mejorar la adherencia a la práctica en diferentes poblaciones, desde atletas de élite hasta pacientes.

Tabla 1

Valores de Data Rate para Diferentes Sensores Biométricos

\begin{tabular}{|l|c|c|}
\hline \multicolumn{1}{|c|}{ SENSOR } & DATA RATE (Akbar et al., 2016) & DATA RATE (Kos et al., 2019) \\
\hline $\begin{array}{l}\text { Ritmo } \\
\text { Cardíaco }\end{array}$ & pocos kbps & $100 \mathrm{kbps}$ \\
\hline Acelerómetro & $35 \mathrm{kbps}$ & $1-200 \mathrm{kbps}$ \\
\hline Giroscopio & $35 \mathrm{kbps}$ & $1-200 \mathrm{kbps}$ \\
\hline SpO2 & - & $100 \mathrm{kbps}$ \\
\hline
\end{tabular}

\section{Materiales y Métodos}

Para este estudio propuesto se especificaron dos tipos de escenarios. El primer escenario con un área rectangular de $42 \mathrm{~m}$ x $57 \mathrm{~m}$ donde se desplazaron los jugadores (nodos) al momento de defender y el segundo escenario de $39 \mathrm{~m}$ x $60 \mathrm{~m}$, al momento de atacar. Se subdividió el área total de cada escenario en subáreas de acuerdo a las posiciones que ocuparon los jugadores tomando en cuenta los mapas de calor de cada uno, y como referencia investigaciones previas, usando un esquema de formación 4-3-3 para defender y 2-4-3 para atacar. Este artículo, basándose en (Külah \& Alemdar, 2020), se propuso la siguiente división mostrada en la Figura 1 y Figura 2. Donde DFC representó a los defensas centrales, DFI y DFD representó a los defensas izquierdo y derecho respectivamente, MC representó a los mediocampistas, EI y ED representaron a los extremos izquierdo y derecho respectivamente y DC representó al delantero centro. Cada nodo se movió dependiendo de la posición del jugador al que representó, basándose en los mapas de calor de cada jugador según (Wehbe et al., 2014), por lo cual se asignaron diferentes colores a estas áreas para poder identificarlos, ya que los nodos no pueden cambiar de área, con el motivo de simplificar el escenario de movilidad de los nodos. Cabe recalcar que existieron posiciones de relevos por lo que en ciertos momentos los nodos o jugadores compartieron la misma área, es por ello que se ha definido áreas conformadas con barras, es el caso de los DFI y EI o DFI y MC. 
Otro aspecto importante tomado en cuenta fue la velocidad de los jugadores (nodos). Por lo cual se consideró velocidades promedios y velocidades máximas donde el entorno cambiaba cada 5 segundos (Antonio Durá Vaquera, n.d.), siendo este el tiempo de movilidad. Después de cumplir este tiempo se cambió la dirección y la velocidad de los nodos utilizando una variable aleatoria uniforme. En (Wehbe et al., 2014) se detallaron las velocidades promedio en $\mathrm{Km} / \mathrm{h}$ y en (Mendez-Villanueva, 2012) y las velocidades máximas, para las distintas posiciones, es decir para defensas, medio-campistas y delanteros. En la Tabla 2 se resumen estos valores.

\section{Figura 1}

Escenario 1 "Equipo Defendiendo"
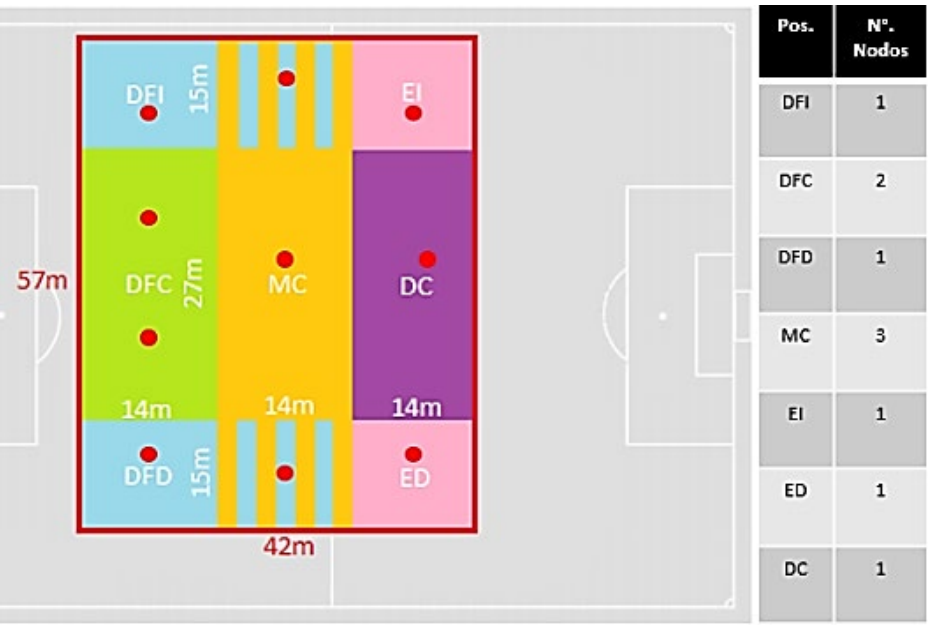

Figura 2

Escenario 2 "Equipo Atacando"

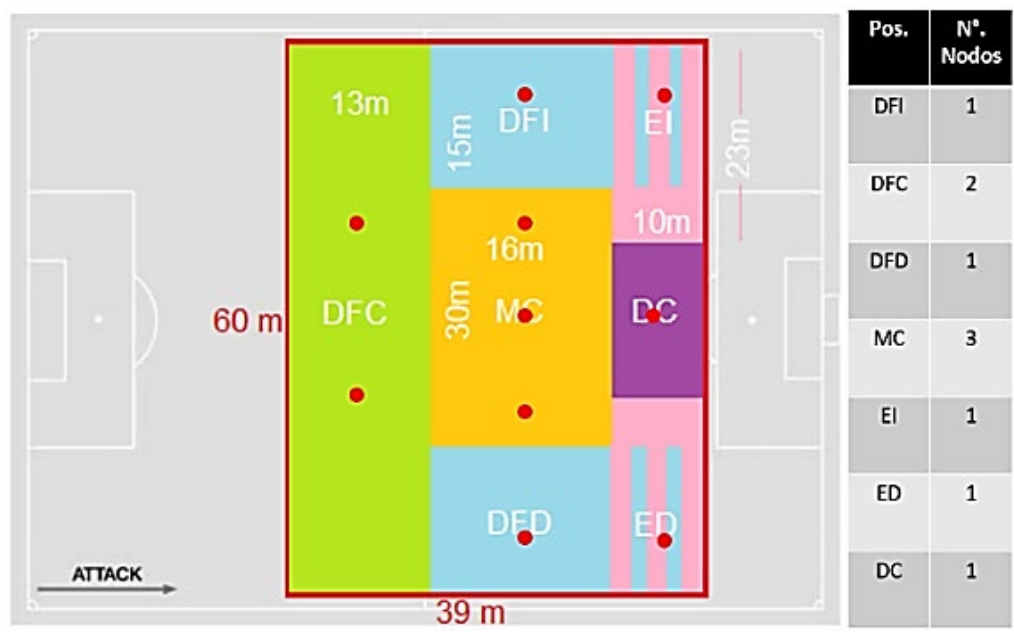

Tabla 2

Velocidades Promedio y Máximas para cada Posición

\begin{tabular}{|l|c|c|c|c|}
\hline & DFC & DFI/DFD & MC & DC \\
\hline Vel. Promedio $[\mathbf{k m} / \mathbf{h}]$ & 6,279 & 6,279 & 6,965 & 6,711 \\
\hline Vel. Max. $[\mathbf{k m} / \mathbf{h}]$ & 28,1 & 26,7 & 27 & 29,1 \\
\hline
\end{tabular}


La metodología utilizada para comparar el desempeño de cada uno de los protocolos de enrutamiento dentro de estos dos escenarios se muestra en la Figura 3. Donde, el primer bloque representó el planteamiento del problema que consistió en encontrar el mejor protocolo de enrutamiento para una red MANET, que transporte datos biométricos procedentes de diferentes sensores colocados en jugadores de fútbol. El segundo bloque permitió definir los protocolos con mejor desempeño y con bases sólidas para la aplicación propuesta, además de ajustar los parámetros de cada uno para mejorar el desempeño.

El siguiente bloque permitió definir las características de los paquetes a transmitir, basándose en la cantidad de información generada por los sensores biométricos. Con ello, se definió el valor de data rate en $100 \mathrm{kbps}$ constantes (CBR), de acuerdo a los valores observados en los trabajos relacionados, ver Tabla 1. De la misma forma se estableció el tamaño del paquete en 100 bytes, debido a que es un valor que estuvo en el rango descrito por los autores (Beitelspacher et al., 2020; Besher et al., 2020; Lloret et al., 2016). Por último, se escogió UDP como protocolo de transporte debido a que comparado con TCP éste es más rápido, simple y eficiente. Esta caracterización permitió generar un tráfico realista entre los nodos.

El cuarto bloque permitió definir las áreas de movilidad de los nodos, así como las velocidades de cada uno dependiendo de la posición que ocupó dentro del campo de juego y el tipo de escenario, para esto se utilizó el módulo Random Walk de NS3 el cual es un modelo de movilidad aleatorio 2D. Hay que tener en cuenta que una de las limitaciones de la simulación es la modelación de la movilidad, modelar el movimiento humano es otro campo de investigación específico (Conti \& Giordano, 2014).

A continuación, se especifican las condiciones de cada escenario estudiado, es decir, el comportamiento de los nodos cuando representan al equipo de fútbol atacando o defendiendo, para lo cual se hizo uso de dos scripts, ya que se definen diferentes áreas en cada uno. Una vez configurados los dos escenarios, se ejecutó la simulación utilizando los protocolos de enrutamiento propuestos y los parámetros de las tarjetas inalámbricas o módulos Wi-fi que trasmiten la información de los sensores biométricos según el tipo de análisis (tres análisis). Algunos de los sensores más comunes utilizaron el estándar IEEE 802.11n como lo hizo en (Hu et al., 2020), por lo que la simulación empleó el mismo. Para generalizar los resultados se hizo uso de una semilla de aleatoriedad y se repitió la simulación utilizando un script de Linux, variando así las condiciones de la simulación. Después, se realizó un análisis estadístico de los resultados obtenidos para cada escenario con cada uno de los protocolos empleados. Finalmente, se establecieron las conclusiones respectivas y se definió el mejor protocolo dependiendo del tipo de métrica. Antes de continuar, se muestran en la Tabla 3 los parámetros utilizados en las simulaciones.

Basándose en la metodología mencionada, se han propuesto tres análisis estadísticos utilizando intervalos de confianza del 95\%, el primero consistió en ejecutar la simulación con un tiempo de 3 minutos o 180 segundos con la configuración de la Tabla 3, usando una potencia fija de $3 \mathrm{dBm}$ y los protocolos de enrutamiento con sus valores por defecto. Se realizaron cinco simulaciones para cada escenario con cada uno de los protocolos de enrutamiento. El segundo análisis consistió en aumentar el número de simulaciones para cada protocolo a 20 , así como el tiempo de cada simulación a 5 minutos o 300 segundos, también se aumentó el intervalo de transmisión a 5, 10, 20, 30 y 60 segundos y se modificaron los archivos de configuración de cada protocolo, para mejorar el desempeño de la red. Por lo tanto, para el protocolo OLSR se acortó el valor del parámetro Hello de 2 segundos a 0.5 segundos, y el parámetro TC se disminuyó de 3 a 1 segundo. Para el protocolo DSDV se cambió el parámetro 
PeriodicUpdateInterval de 15 a 2 segundos, con esto se redujo el periodo de intercambio de tablas de enrutamiento completas entre nodos.

El siguiente parámetro modificado fue el SettlingTime, reduciendo el tiempo mínimo de almacenamiento de una actualización de 5 a 3 segundos. Ahora, para AODV el primer parámetro que se modificó fue el intervalo Hello, el cual cambió de 1 a 0.5 segundos. También se estableció al parámetro ActiveRouteTimeout en 2 segundos en lugar del valor original de 3 segundos, este parámetro determinó el periodo de tiempo durante el cual una ruta es considerada como válida, por lo tanto, al tener un escenario con alta movilidad se disminuyó este periodo.

En el tercer análisis se escogieron los dos protocolos con mejor rendimiento que en el segundo análisis y con el intervalo de tiempo más óptimo. Además, varió la potencia del transmisor en el rango mostrado en la Tabla 3. Cabe destacar que se mantuvo las modificaciones realizadas a los protocolos de enrutamiento en el segundo análisis.

Figura 3

Resumen de la Metodología Aplicada

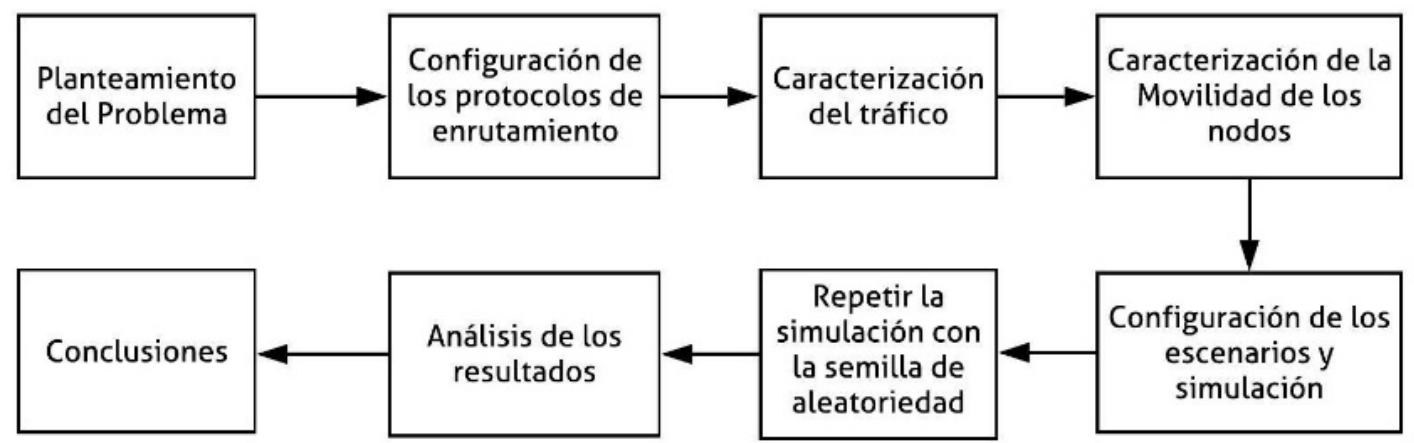

Tabla 3

Parámetros de la Simulación

\begin{tabular}{|c|c|c|c|}
\hline PARÁMETROS & ANÁLISIS 1 & ANÁLISIS 2 & ANÁLISIS 3 \\
\hline Estándar WI-FI & \multicolumn{3}{|c|}{ IEEE $802.11 \mathrm{n}(2.4 \mathrm{GHz})$} \\
\hline Capa Física & \multicolumn{3}{|c|}{ ErpOfdmRate48Mbps } \\
\hline Protocolo de transporte & \multicolumn{3}{|c|}{ UDP } \\
\hline Tipo de tráfico & \multicolumn{3}{|c|}{ CBR } \\
\hline Tamaño del paquete & \multicolumn{3}{|c|}{100 bytes } \\
\hline Data Rate & \multicolumn{3}{|c|}{$100 \mathrm{kbps}$} \\
\hline Tipo de Movilidad & \multicolumn{3}{|c|}{ Random Walk } \\
\hline Tiempo de Movilidad & \multicolumn{3}{|c|}{5 seg } \\
\hline Tiempo de Simulación & $180 \mathrm{seg}$ & $300 \mathrm{seg}$ & $300 \mathrm{seg}$ \\
\hline Intervalo de Transmisión & $1 \mathrm{seg}$ & $5,10,20,30$ y $60 \mathrm{seg}$ & $20 \mathrm{seg}$ \\
\hline $\begin{array}{l}\text { Número de Simulaciones } \\
\text { por Protocolo }\end{array}$ & 5 & 20 & 20 \\
\hline Potencia del Transmisor & $3 \mathrm{dBm}$ & $3 \mathrm{dBm}$ & $0-10 \mathrm{dBm}$ \\
\hline
\end{tabular}


Cabe señalar que la cantidad de nodos y el tipo de escenario demandó un tiempo considerable para realizar múltiples simulaciones, debido a la alta carga computacional requerida. Con el objetivo de realizar el estudio con varias pruebas, 230 en total, se utilizaron tiempos de 3 y 5 minutos, ya que incrementar estos tiempos es una limitación debido a las demoras que requieren cada uno de los estudios. Entonces, se prefirió realizar múltiples simulaciones de corta duración para obtener resultados que se puedan valorar estadísticamente, en lugar de una sola simulación de larga duración.

\section{Primer análisis}

\section{Resultados y Discusión}

En la Figura 4 se presentan los resultados obtenidos para el PDR en el escenario 1 y escenario 2. Es evidente que la mejor tasa de recepción de paquete se logra con el protocolo de enrutamiento reactivo AODV, llegando hasta un $49 \%$ de paquetes recibidos. Mientras que el peor desempeño lo obtiene DSDV con tan solo un 27\%. Sin embargo, los intervalos de confianza son mayores en AODV, lo que implica que hay más variaciones entre simulaciones. En cuanto al delay promedio obtenido para cada protocolo, se pueden observar los resultados logrados en la Figura 5, que el protocolo OLSR alcanza los mejores resultados ya que tienen el menor delay. En cambio, los otros dos protocolos no varían mucho, pero se tienen que tomar en cuenta que para el escenario 2 el protocolo DSDV tiene intervalos de confianza muy amplios. Finalmente, se puede observar en la Figura 6, que AODV tiene el mejor throughput promedio en los dos escenarios, le sigue el protocolo OLSR y el protocolo AODV en este caso es el peor.

\section{Figura 4}

\section{Comparativa del PDR para cada Protocolo de Enrutamiento}

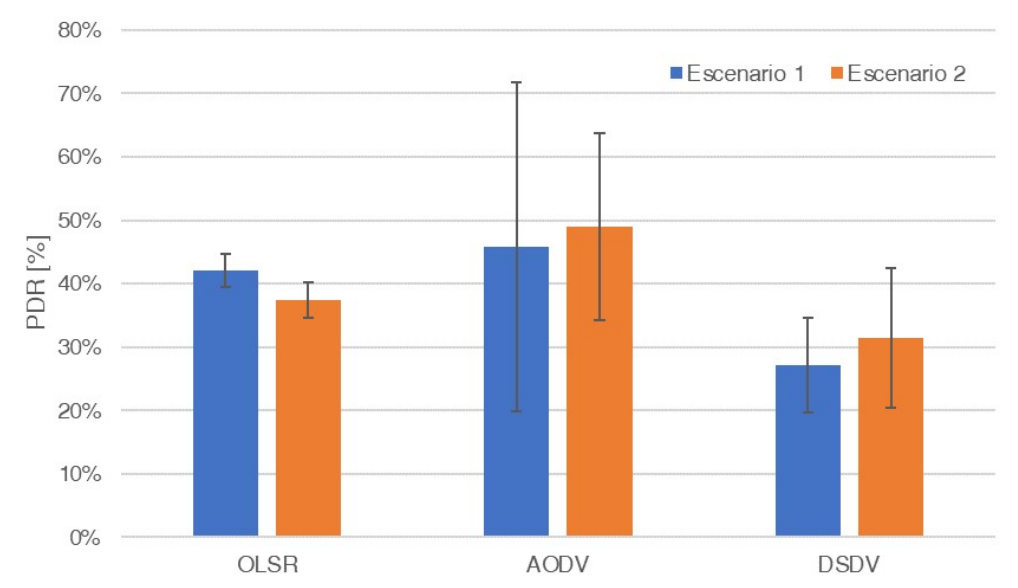

Al analizar las métricas se observa que el protocolo AODV tiene una alta variación en los intervalos de confianza. Por lo que depende mucho de las condiciones de movilidad, velocidad y del canal, para tener un buen desempeño. En ciertos instantes el tráfico cae prácticamente a cero, debido a la perdida de paquetes. Por este motivo se verifica las tablas de enrutamiento y las posiciones de los nodos en estos instantes y se verifica que, debido al movimiento de los nodos, existen instantes en los que un nodo sale del rango de cobertura con algún nodo vecino pero las tablas de enrutamiento tardan un determinado tiempo en converger nuevamente, en el caso de OLSR, se escoge un intervalo de 3 segundos para intercambiar mensajes TC. Además, se puede notar que debido a la naturaleza del protocolo AODV, este resulta ser más eficiente debido a que actualiza sus tablas de enrutamiento únicamente cuando va a transmitir tráfico. 


\section{Figura 5}

Comparativa del Delay para cada Protocolo de Enrutamiento

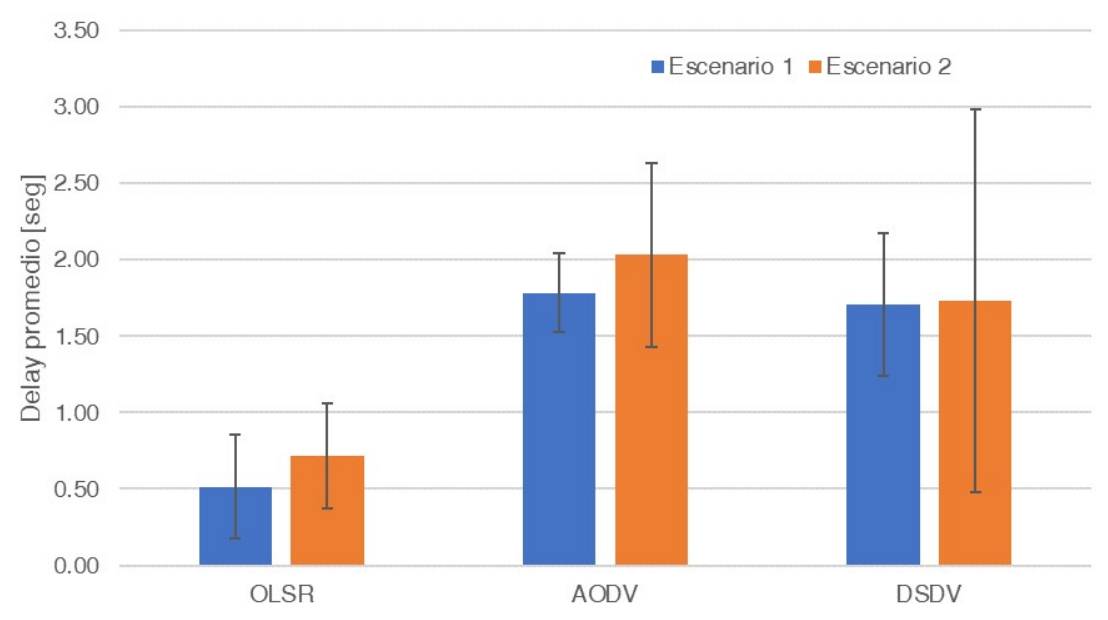

Figura 6

Comparativa del Throughput Promedio para cada Protocolo de Enrutamiento

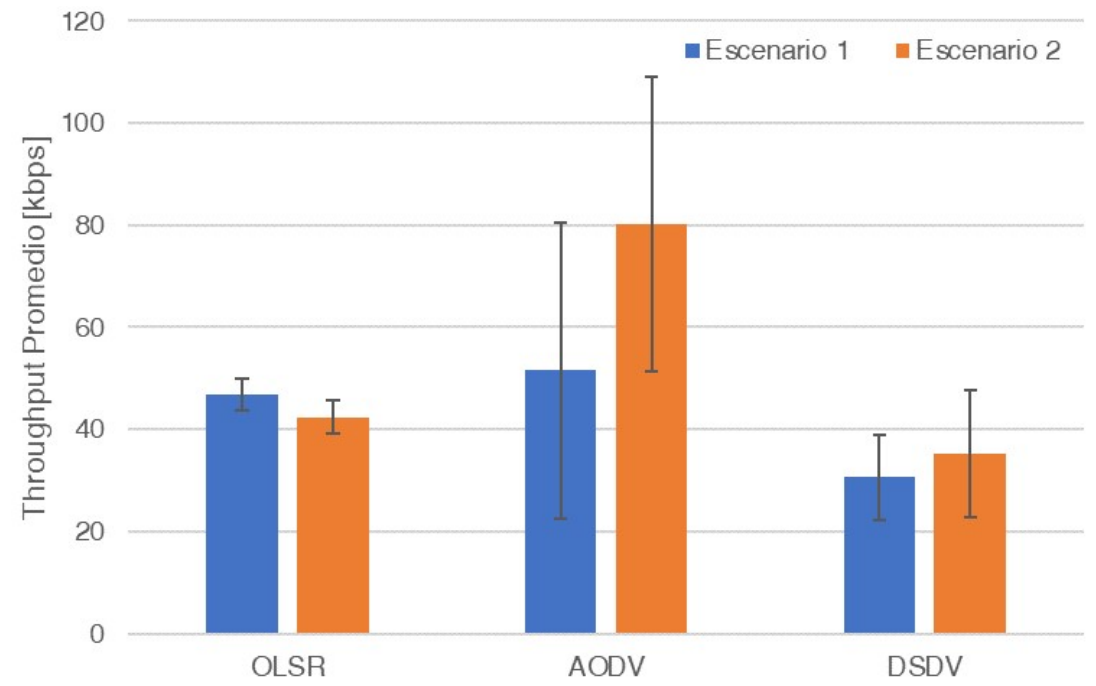

\section{Segundo análisis}

Los resultados muestran que, al aumentar el número de simulaciones y ajustar los parámetros de los protocolos, las métricas mejoraron notablemente con respecto a los resultados anteriores. Esto se debe a que los protocolos de enrutamiento tienen más tiempo para converger y por lo tanto existe una menor pérdida de paquetes, además debido a la movilidad de los nodos y al canal inalámbrico existen interferencias, pérdidas de paquetes y por lo tanto el PDR no supera el $70 \%$. Además, los intervalos de confianza se redujeron considerablemente, lo cual indica que existe una menor desviación entre los valores. En la Figura 7, se observa que el PDR tiene una tendencia creciente conforme se incrementa el intervalo, sin embargo, este crecimiento no es tan acelerado, por lo que no hay gran diferencia a partir de los 20 segundos. Con respecto a los protocolos se puede notar que AODV presenta mejores resultados en el escenario 1 y DSDV para el escenario 2. 
Figura 7

Comparativa del PDR para cada Protocolo de Enrutamiento

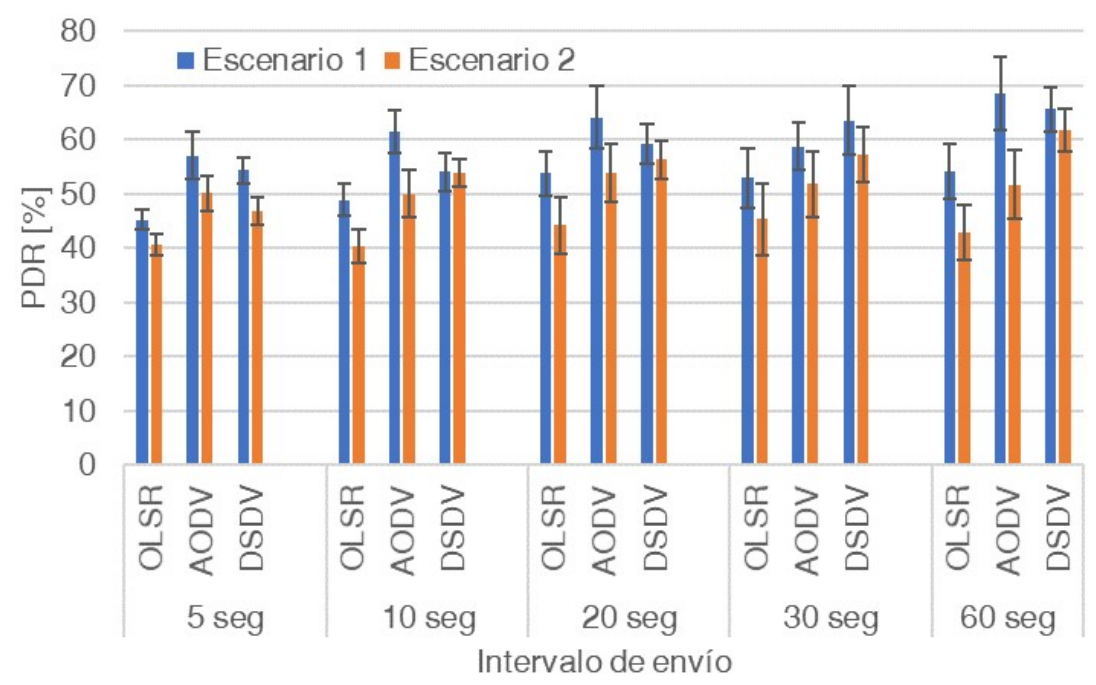

Figura 8

Comparativa del Delay para cada Protocolo de Enrutamiento

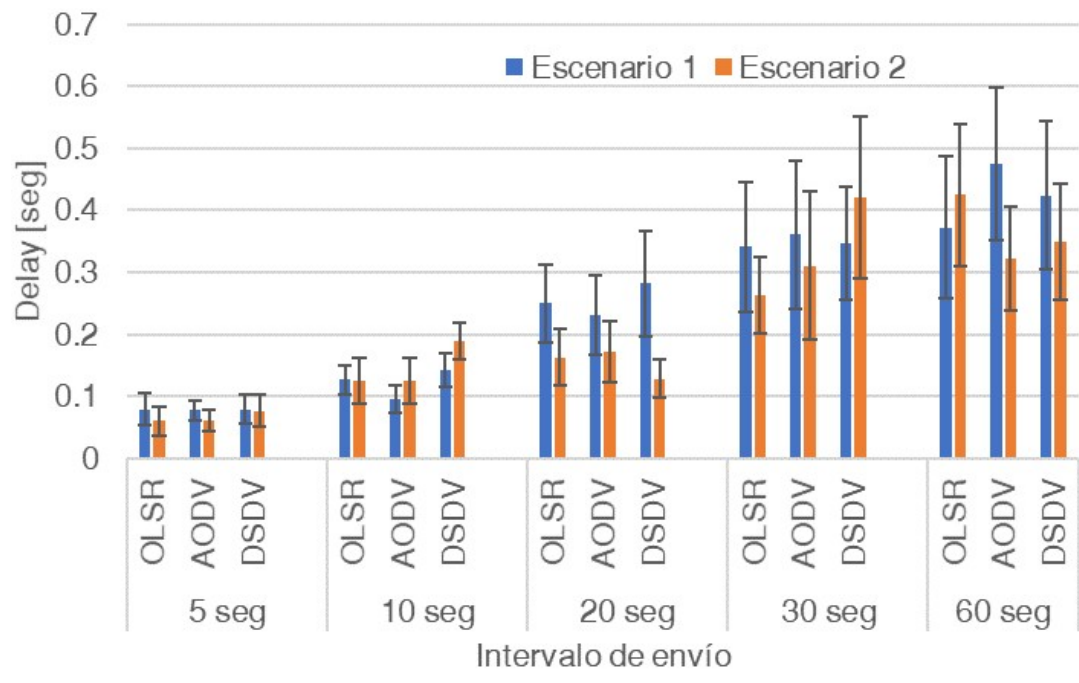

En cuanto al delay, la Figura 8 muestra que el delay aumenta rápidamente conforme se incrementa el intervalo de transmisión, pero al comparar los protocolos de enrutamiento no se evidencia una diferencia muy marcada entre cada uno. Se puede observar que a partir de los 20 segundos los intervalos de confianza crecen debido a que al separar las transmisiones disminuye la cantidad de información recibida y por lo tanto un error tendrá un mayor impacto en los resultados. Con respecto a la última métrica, el throughput, en la Figura 9 indica que el mejor protocolo es AODV para el escenario 1 y el protocolo DSDV para el escenario 2. Además, se puedo observar que el throughput no alcanza los $100 \mathrm{kbps}$ que es la tasa fijada en cada uno de los trasmisores, por el contrario tienen un valor promedio de $67.82 \mathrm{kbps}$ y 58.96 kbps para el escenario 1 y 2 respectivamente, esto ocurre debido a las características propias de una red ad-hoc multi-salto, ya que el throughput disminuye con cada salto según (González et al., 2016). 
Figura 9

Comparativa del Throughput para cada Protocolo de Enrutamiento

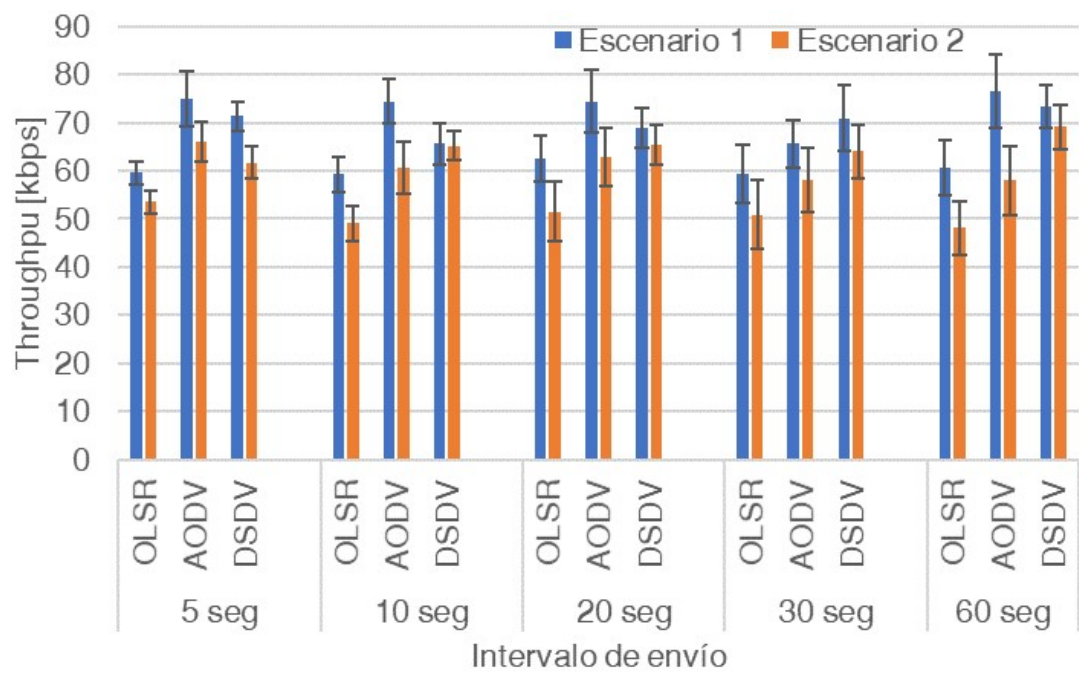

\section{Tercer análisis}

De acuerdo a los resultados anteriores, se descarta el protocolo OLSR debido a que tiene el peor rendimiento comparado con los otros dos protocolos en la mayoría de métricas y escenarios. Además, se establece el intervalo de transmisión en 20 segundos ya que se obtienen mejores resultados y permite la convergencia de los protocolos de enrutamiento. También se incrementa la potencia del transmisor, y se puede notar que las métricas mejoran su rendimiento con respecto a los resultados de la sección anterior. Sin embargo, a partir de los 4 dBm la red Ad Hoc deja de ser multi-salto y pasa a conformar una red MESH. En la Figura 10, conforme aumenta la potencia, también aumenta el PDR debido a que se disminuye la cantidad de saltos, sin embargo, también aumentan las interferencias por lo que todavía existen pérdidas de paquetes. Por otro lado, en el escenario 1 el protocolo AODV es mejor que DSDV hasta los $4 \mathrm{dBm}$ o, en otras palabras, AODV tiene un mejor rendimiento que DSDV en la red multisalto, pero DSDV es mejor cuando la red pasa a ser de tipo MESH. Al analizar el escenario 2, se observa que DSDV es mejor que AODV sin importar el valor de la potencia del transmisor.

Figura 10

\section{Comparativa del PDR para cada Protocolo de Enrutamiento}

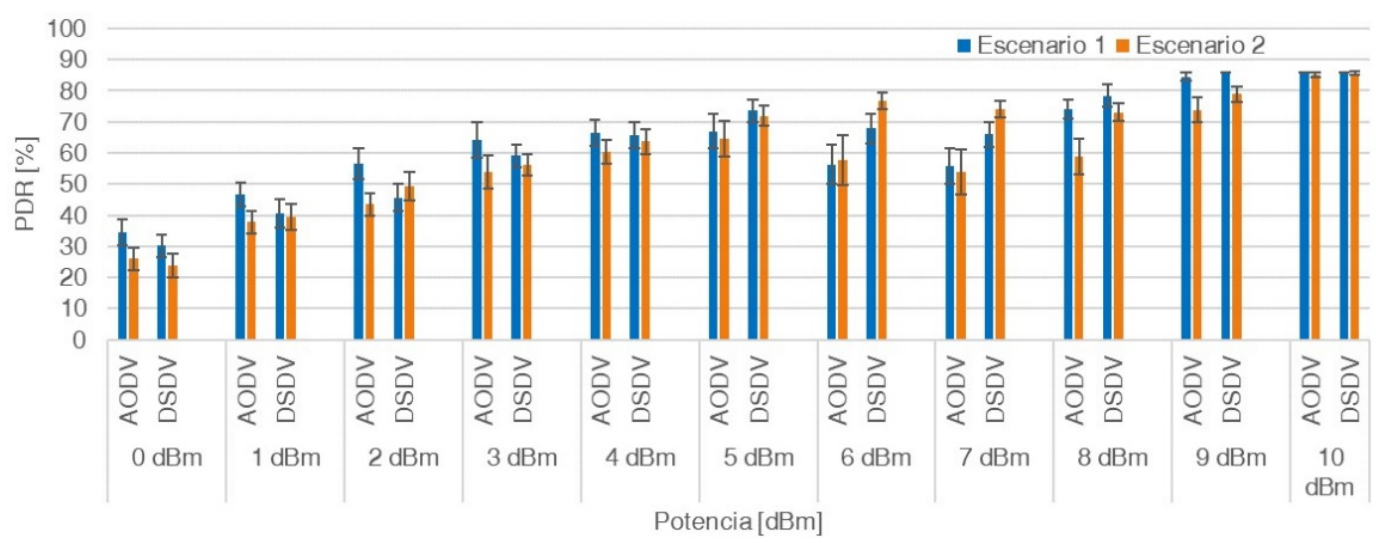

En la Figura 11, se puede notar que el protocolo AODV tiene un mayor delay que DSDV en los dos escenarios, esto se debe a que AODV al ser un protocolo reactivo le toma mayor tiempo obtener la información de ruteo de los demás nodos. También se observa que el menor delay en una red MESH se obtiene con una potencia de $10 \mathrm{dBm}$, mientras que en una 
red multi-salto el mejor resultado se logra con $3 \mathrm{dBm}$, de lo cual se puede notar que en ambos tipos de red el mejor resultado se obtiene con el máximo de potencia. Con respecto al throughput, la Figura 12 indica que el valor incrementa conforme aumenta el nivel de potencia de los transmisores. También se nota que en el escenario 1, AODV es mejor que DSDV hasta los $4 \mathrm{dBm}$, y además se observa que con $10 \mathrm{dBm}$ no existe una diferencia notable, teniendo además un error menor al 1\%. Ahora, en el escenario 2 DSDV siempre es mejor que AODV a excepción de los $10 \mathrm{dBm}$, donde nuevamente no hay una diferencia significativa. Finalmente, se puede notar que con $10 \mathrm{dBm}$ el throughput coincide con el data rate de transmisión configurado inicialmente, siendo así el mejor resultado posible para esta métrica.

Figura 11

Comparativa del Delay para cada Protocolo de Enrutamiento

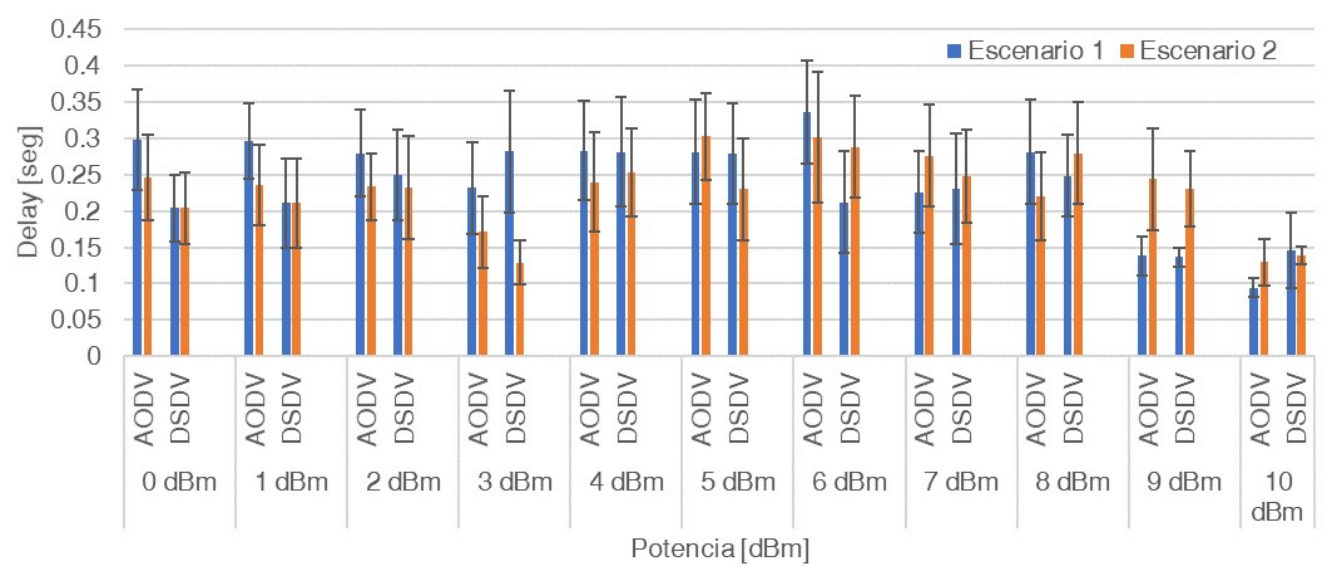

Figura 12

Comparativa del Throughput para cada Protocolo de Enrutamiento

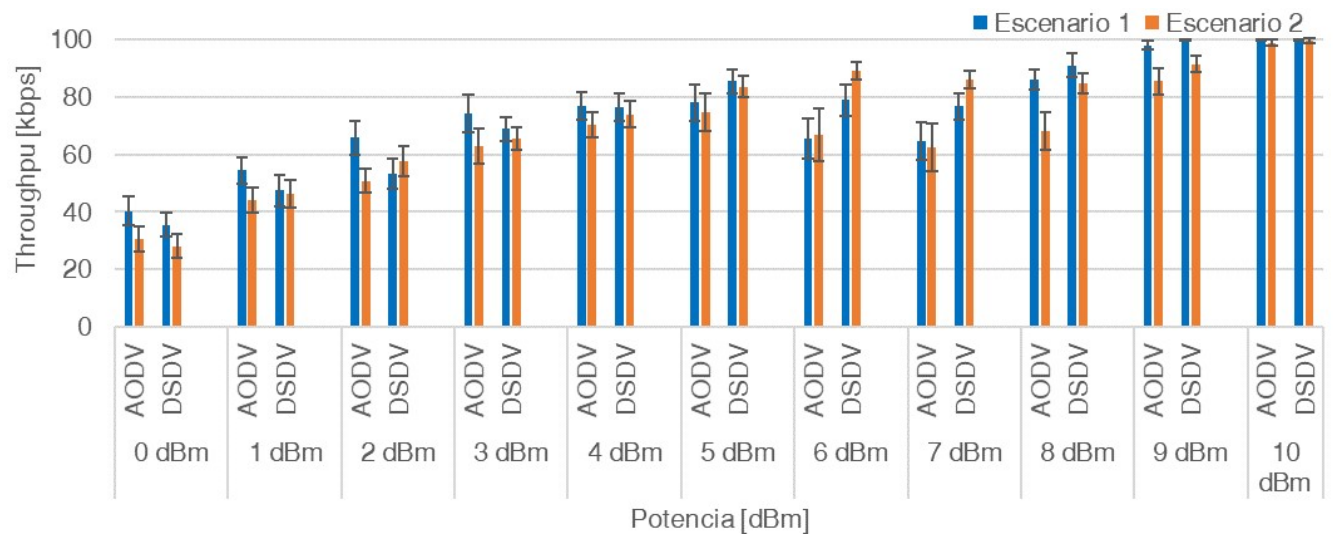

\section{Conclusiones}

En el primer análisis, se puede concluir que el protocolo de enrutamiento reactivo AODV tiene un mejor desempeño en las tres métricas analizadas (PDR, delay y throughput), pero con unos intervalos de confianza muy amplios. Se comprueba que el número máximo de saltos generados fue de tres, cuando el nodo se encontraba muy alejado del nodo receptor. Mientras que cuando el nodo transmisor se encontraba más central o cerca del nodo receptor el número de saltos generado era de uno. De acuerdo al perfil del tráfico obtenido y el PDR para cada uno de los protocolos, se puede notar que existe una gran pérdida de paquetes. Estas pérdidas ocurren debido al comportamiento aleatorio de los nodos, lo cual genera cambios en la topología de la red. Es decir, las tablas de enrutamiento se actualizan constantemente, pero 
los movimientos bruscos de los nodos (jugadores) no permiten que converjan en ciertos instantes. Además, se comprueba que el crecimiento del throughput afecta la efectividad del envío de paquetes debido a las limitaciones del ancho de banda.

En el segundo análisis las tres métricas mejoran y existe una reducción de los intervalos de confianza debido al cambio en los parámetros de cada protocolo, el espaciamiento entre transmisiones y por el incremento del número de simulaciones. Por ejemplo, con un espaciamiento de 20 segundos el delay se reduce un $64.58 \%$ para OLSR, $89.36 \%$ para AODV y $88.31 \%$ para DSDV, realizando un promedio entre los dos escenarios. Por otra parte, al aumentar el intervalo de transmisión se incrementa el delay de cada paquete, por lo que se escoge 20 segundos como un valor óptimo. Con este valor el throughput aumenta un $27.33 \%$ para OLSR, un 105\% para DSDV realizando el promedio entre los dos escenarios. Mientras que para AODV el throughput aumenta un $44.33 \%$ en el escenario 1. Además, se evidencia que en el escenario 1 (Defensa) el protocolo con mejor rendimiento es AODV y para el escenario 2 (Ataque) es DSDV. Finalmente, en el análisis tres, mejoran los resultados en los dos escenarios. Sin embargo, al superar los $4 \mathrm{dBm}$ en la potencia del transmisor, la red se convierte en una red MESH. Y, en definitiva, se comprueba que los dos protocolos no tienen gran diferencia en su desempeño en las tres métricas con una potencia de $10 \mathrm{dBm}$, por lo que se puede escoger cualquiera de los dos para este tipo de escenarios.

\section{Reconocimientos}

Los autores de este trabajo agradecen al Departamento de Eléctrica, Electrónica y Telecomunicaciones de la Universidad de Cuenca por todo el apoyo brindado y herramientas proporcionadas para la elaboración de este artículo.

\section{Referencias}

Akbar, M. S., Yu, H., \& Cang, S. (2016). Delay, Reliability, and Throughput Based QoS Profile: A MAC Layer Performance Optimization Mechanism for Biomedical Applications in Wireless Body Area Sensor Networks. Journal of Sensors, 2016. https://doi.org/10.1155/2016/7170943

Al-Dhief, F. T., Sabri, N., Salim, M. S., Fouad, S., \& Aljunid, S. A. (2018). MANET Routing Protocols Evaluation: AODV, DSR and DSDV Perspective. MATEC Web of Conferences, 150, 06024. https://doi.org/10.1051/matecconf/201815006024

Antonio Durá Vaquera, J. (n.d.). Revisión Bibliográfica: Entrenamiento SAQ (speed, agility, quickness) en fútbol.

Bai, Y., Mai, Y., \& Wang, N. (2017, June 7). Performance comparison and evaluation of the proactive and reactive routing protocols for MANETs. Wireless Telecommunications Symposium. https://doi.org/10.1109/WTS.2017.7943538

Beitelspacher, S., Besher, K. M., \& Zamshed Ali, M. (2020, June 1). Sensor Driven Priority Routing of Health Care Data Packet in IoT Network. IEEE World Forum on Internet of Things, WF-IoT 2020 Symposium Proceedings. https://doi.org/10.1109/WF-IoT48130.2020.9221478

Besher, K. M., Beitelspacher, S., Nieto-Hipolito, J. I., \& Ali, M. Z. (2020). Sensor Initiated Healthcare Packet Priority in Congested IoT Networks. IEEE Sensors Journal, 1-1. https://doi.org/10.1109/jsen.2020.3012519

Campanile, L., Gribaudo, M., Iacono, M., Marulli, F., \& Mastroianni, M. (2020). Computer network simulation with ns-3: A systematic literature review. Electronics (Switzerland), 9(2), 1-25. https://doi.org/10.3390/electronics9020272

Conti, M., \& Giordano, S. (2014). Mobile ad hoc networking: Milestones, challenges, and new research 
directions. IEEE Communications Magazine, 52(1), 85-96. https://doi.org/10.1109/MCOM.2014.6710069

Gamess, E., \& Russoniello, A. (2018). Evaluation of Different Routing Protocols for Mobile Ad-Hoc Networks in Scenarios with High-Speed Mobility. I. J. Computer Network and Information Security, 10, 46-52. https://doi.org/10.5815/ijcnis.2018.10.06

González, S., Castellanos, W., Guzmán, P., Arce, P., \& Guerri, J. C. (2016). Simulation and experimental testbed for adaptive video streaming in ad hoc networks. Ad Hoc Networks, 52, 89-105. https://doi.org/10.1016/j.adhoc.2016.07.007

Hu, J., Wang, J., \& Xie, H. (2020). Wearable bracelets with variable sampling frequency for measuring multiple physiological parameter of human. Computer Communications, 161, 257-265. https://doi.org/10.1016/J.COMCOM.2020.07.043

Kos, A., Milutinović, V., \& Umek, A. (2019). Challenges in wireless communication for connected sensors and wearable devices used in sport biofeedback applications. Future Generation Computer Systems, 92, 582-592. https://doi.org/10.1016/j.future.2018.03.032

Külah, E., \& Alemdar, H. (2020). Quantifying the value of sprints in elite football using spatial cohesive networks. Chaos, Solitons and Fractals, 139, 110306. https://doi.org/10.1016/j.chaos.2020.110306

Kurniawan, A., Kristalina, P., \& Hadi, M. Z. S. (2020). Performance Analysis of Routing Protocols AODV, OLSR and DSDV on MANET using NS3. IES 2020 - International Electronics Symposium: The Role of Autonomous and Intelligent Systems for Human Life and Comfort, 199-206. https://doi.org/10.1109/IES50839.2020.9231690

Lamaarti, F., Arafsha, F., Hafidh, B., \& El Saddik, A. (2019). Automated Athlete Haptic Training System for Soccer Sprinting. Proceedings - 2nd International Conference on Multimedia Information Processing and Retrieval, MIPR 2019, 303-309. https://doi.org/10.1109/MIPR.2019.00061

Li, R. T., Kling, S. R., Salata, M. J., Cupp, S. A., Sheehan, J., \& Voos, J. E. (2016). Wearable Performance Devices in Sports Medicine. In Sports Health (Vol. 8, Issue 1, pp. 74-78). SAGE Publications Inc. https://doi.org/10.1177/1941738115616917

Li, S., Zhang, B., Fei, P., Shakeel, P. M., \& Samuel, R. D. J. (2020). Computational efficient wearable sensor network health monitoring system for sports athletics using IoT. In Aggression and Violent Behavior (p. 101541). Elsevier Ltd. https://doi.org/10.1016/j.avb.2020.101541

Lloret, J., Garcia, M., Catala, A., \& Rodrigues, J. J. P. C. (2016). A group-based wireless body sensors network using energy harvesting for soccer team monitoring. International Journal of Sensor Networks, 21(4), 208-225. https://doi.org/10.1504/IJSNET.2016.079172

Massard, T., Eggers, T., \& Lovell, R. (2018). Peak speed determination in football: is sprint testing necessary? Science and Medicine in Football, 2(2), 123-126. https://doi.org/10.1080/24733938.2017.1398409

Mendez-Villanueva, A. (2012). Repeated High-Speed Activities during Youth Soccer Games in Relation to Changes in Maximal Sprinting and Aerobic Speeds. Article in International Journal of Sports Medicine. https://doi.org/10.1055/s-0032-1316363

Modric, T., Versic, S., \& Sekulic, D. (2020). Aerobic fitness and game performance indicators in professional football players; playing position specifics and associations. Heliyon, 6(11), e05427. https://doi.org/10.1016/j.heliyon.2020.e05427

Pappalardo, L., Cintia, P., Ferragina, P., Massucco, E., Pedreschi, D., \& Giannotti, F. (2019). PlayeRank: Datadriven performance evaluation and player ranking in soccer via a machine learning approach. $A C M$ Transactions on Intelligent Systems and Technology, 10(5), 59. https://doi.org/10.1145/3343172

Sharma, M. S., \& Shruti Thapar, M. (n.d.). Comparative Performance Analysis of AODV, DSDV and OLSR Routing Protocols in MANET Using OPNET. In International Journal of Novel Research in Computer Science and Software Engineering (Vol. 2). Retrieved May 12, 2021, from www.noveltyjournals.com 
Singh, K., \& Verma, A. K. (2015, August 26). Experimental analysis of AODV, DSDV and OLSR routing protocol for flying adhoc networks (FANETs). Proceedings of 2015 IEEE International Conference on Electrical, Computer and Communication Technologies, ICECCT 2015. https://doi.org/10.1109/ICECCT.2015.7226085

Wehbe, G. M., Hartwig, T. B., \& Duncan, C. S. (2014). Movement analysis of australian national league soccer players using global positioning system technology. Journal of Strength and Conditioning Research, 28(3), 834-842. https://doi.org/10.1519/JSC.0b013e3182a35dd1

Yefa Mai, Yuxia Bai, \& Nan Wang. (2017). Performance Comparison and Evaluation of the Routing Protocols for MANETs Using NS3. J. of Electrical Engineering, 5(4). https://doi.org/10.17265/2328$2223 / 2017.04 .003$ 